\title{
The most sensitive SETI observation by multi-beam coincidence matching strategy towards exoplanet systems
}

\section{Zhen-zhao Tao}

Beijing Normal University

Hai-chen Zhao

Beijing Normal University https://orcid.org/0000-0002-5485-1877

\section{Zhi-song Zhang}

National Astronomical Observatories, Chinese Academy of Sciences

Tong-Jie Zhang ( $\nabla$ tjzhang@bnu.edu.cn )

Beijing Normal University https://orcid.org/0000-0002-3363-9965

Vishal Gajjar

University of California, Berkeley https://orcid.org/0000-0002-8604-106X

\section{Yan Zhu}

National Astronomical Observatories, Chinese Academy of Sciences

\section{You-ling Yue}

National Astronomical Observatories, Chinese Academy of Sciences

Hai-yan Zhang

National Astronomical Observatories, Chinese Academy of Sciences

Wen-fei Liu

Qilu Normal University

\section{Shi-yu Li}

Beijing Planetarium, Beijing Academy of Science and Technology

Jian-chen Zhang

Dezhou University

\section{Cong Liu}

Dezhou University

\section{Hong-feng Wang}

Dezhou University

\section{Ran Duan}

National Astronomical Observatories, Chinese Academy of Sciences

\section{Lei Qian}

National Astronomical Observatories, Chinese Academy of Sciences

Cheng-jin Jin 
National Astronomical Observatories, Chinese Academy of Sciences

\section{Di Li}

National Astronomical Observatories, Chinese Academy of Sciences https://orcid.org/0000-00033010-7661

\section{Andrew Siemion}

University of California, Berkeley

\section{Peng Jiang}

National Astronomical Observatories

\section{Dan Werthimer}

University of California Berkeley

\section{Jeff Cobb}

University of California Berkeley

\section{Eric Korpela}

University of California Berkeley https://orcid.org/0000-0001-8078-9395

\section{David Anderson}

University of California Berkeley

\section{Article}

\section{Keywords:}

Posted Date: February 22nd, 2022

DOI: https://doi.org/10.21203/rs.3.rs-1335086/v1

License: (c) (i) This work is licensed under a Creative Commons Attribution 4.0 International License. Read Full License 


\section{The most sensitive SETI observation by multi-beam coinci- dence matching strategy towards exoplanet systems}

3 Z. Z. Tao ${ }^{1,2}$; H. C. Zhao

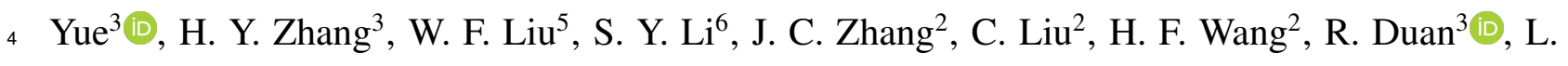

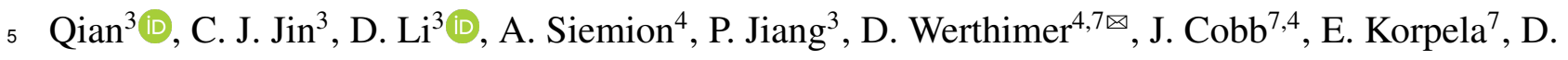
6 P. Anderson ${ }^{7}$,

${ }_{7}^{1}$ Department of Astronomy,Beijing Normal University,Beijing, China

${ }_{8}^{2}$ Institute for Astronomical Science, Dezhou University, Dezhou, China

${ }_{9}^{3}$ National Astronomical Observatories, Chinese Academy of Sciences, Beijing, China

${ }_{10}{ }^{4}$ Breakthrough Listen, University of California Berkeley, Berkeley, CA, USA

${ }_{11}{ }^{5}$ College of Physics and Electronic Engineering, Qilu Normal University, Jinan, China

${ }_{12}{ }^{6}$ Beijing Planetarium, Beijing Academy of Science and Technology, Beijing, China

${ }_{13}{ }^{7}$ Space Sciences Laboratory, University of California Berkeley, Berkeley, CA, USA

'Are we alone?' The Search for Extra-Terrestrial Intelligence (SETI) aims to answer this profound question. Apart from examining environments in our solar system and detecting biosignatures in exoplanet atmospheres ${ }^{1}$, SETI is another main approach to search for life beyond Earth by detecting technosignatures indicative of extra-terrestrial intelligence (ETI) ${ }^{2 / 3}$, such as engineering radio signals. Massive efforts have been made by SETI scientists using

Email:tjzhang@bnu.edu.cn, orcid.org/0000-0002-3363-9965

Email:vishalg@berkeley.edu, orcid.org/0000-0002-8604-106X

Email:danw@ssl.berkeley.edu

*These authors contributed equally to this work. 
radio telescopes around the world ${ }^{4-17}$. Though some candidate signals were detected, none of them has been confirmed as an ETI technosignature so far. Most targeted SETI observations performed in recent years use on-off strategy to distinguish signals transmitted from celestial bodies from radio frequency interference (RFI) generated near the ground. Here we report a SETI campaign employing another SETI observation strategy, multi-beam coincidence matching (MBCM), at the Five-hundred-meter Aperture Spherical radio Telescope (FAST) towards 33 currently discovered planetary systems, searching for narrow band drifting signals at a band of $1.05-1.45$ GHz. Our observations achieve an unprecedented sensitivity with a minimum Equivalent Isotropic Radiated Power (EIRP) of $1.5 \times 10^{9}$ W. We process the data of two orthogonal polarization separately, aiming to discriminate instrumental RFI signals. A particular signal at $1140.604 \mathrm{MHz}$ from the observation towards Kepler-438 passes our initial selection criteria. Although we have not yet determined the exact cause of this signal, its polarization characteristic suggests that it is most likely to be attributed to RFI. In spite of this, our work verifies that compared to single-beam on-off strategy, MBCM greatly improves both time efficiency and radio frequency interference (RFI) identification effectiveness of targeted SETI observations.

With an enormous collecting area (illuminated aperture of $300 \mathrm{~m}$ ), a large sky region coverage $\left(-14^{\circ}\right.$ to $+66^{\circ}$ in declination) and the cryogenically-cooled L-band 19-beam receiver (system temperature of $\sim 20 \mathrm{~K}$ ), FAST ${ }^{18-20}$ is well positioned to conduct highly sensitive and efficient searches for ETI technosignatures ${ }^{21}$, and SETI is one of the five key science goals specified in the original FAST project plan 22 . In 2019, the first commensal SETI survey by FAST was per- 
formed and two groups of candidate signals were detected ${ }^{23}$. Here in this work, we present the first targeted SETI observations by FAST. From 2020 November to 2021 September, 33 currently discovered planetary systems have been observed (Fig. 5), including 29 systems hosting planets in their habitable zones ${ }^{24 \sqrt{26}}$ and 5 systems in the Earth transit zone $e^{27}$, namely, worlds that resemble ours and worlds that can see us.

The greatest challenge for SETI observations is RFI identification and excision ${ }^{23}$. In recent years, targeted SETI observations adopt on-off strategy for this purpose $\frac{10[13}{17}$, alternating the telescope pointing between a target (on-source) and several reference locations (off-source). The angular distance between an on-source and an off-source should be at least several times the full width at half maximum (FWHM), of the telescope, thus ensuring signals transmitted from the on-source cannot be detected from the off-source. Ubiquitous RFI can enter the side lobes of the beam, so it can be detected in both on- and off-observations. Hence, signals detected from both the on-source and any of the off-sources are identified as RFI and are removed directly, while the remaining filtered signals will be further examined.

However, on-off strategy is inefficient for FAST because of its lengthy slewing time, which costs $\sim 10$ min per pointing $\mathrm{g}^{20}$. Thanks to the FAST L-band 19-beam receiver $\frac{18+20}{20}$, the observation efficiency can be improved significantly by multi-beam observation. Based on the principles of on-off strategy, we design the multi-beam coincidence matching strategy for targeted SETI observations by FAST. During an observation, all 19 beams on the receiver record data simultaneously while beam 1 points to the target. The angular distance between two adjacent beams is $\lesssim 6^{\prime}$, about 
twice the L-band FWHM (2.9'). In addition to beam 1, signals transmitted from the target are likely to cover several of the 6 beams adjacent to beam 1, but are impossible to cover the 6 outermost beams (beam 8, 10, 12, 14, 16 and 18), which are 4 FWHM from beam 1. Therefore, these 6 beams serve as reference locations like the off-sources used in on-off strategy, and signals detected by both beam 1 and any of these 6 beams are rejected directly (Fig.1 1). Using as many as 6 reference beams can effectively reduce the contingency caused by low $\mathrm{S} / \mathrm{N}$ and signal incident direction.

The preliminary screening of signals only uses the data of these 7 beams, but can remove the vast majority of signals detected by beam 1 . Then we require the data of other 12 beams to check the beam coverage of the remaining signals. We stipulate that an extra-terrestrial signal cannot cover two beams that are separated by one or more beams, otherwise it is RFI. This criterion can also be used to conduct blind search in the sky region around observed targets, making the most of limited observation time. In other words, every beam on the 19-beam receiver can be considered as an on-source. For example, as shown in Fig.1p, a signal detected by beam 5, 6, 15 and 16 is permitted because no two beams are separated by a complete beam. By contrast, a signal with a beam coverage like Fig.1c should be rejected since beam 5 and beam 17 are separated by beam 6 .

Apart from high time efficiency, MBCM overcomes a crucial drawback of on-off strategy. On-off strategy cannot identify RFI signals that happen to be generated when observing on-source and interrupted when observing off-source, such as signals with a duty cycle matching the onoff cadence ${ }^{28}$. Since MBCM observes a target and reference locations simultaneously, such time 


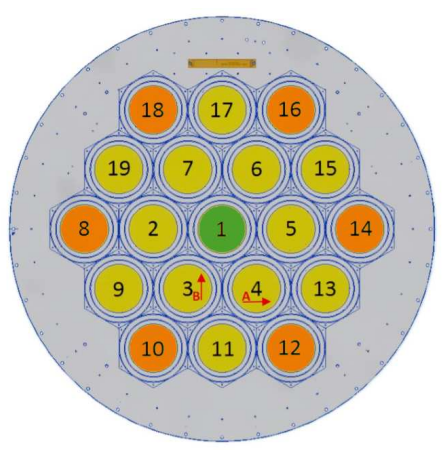

(a)

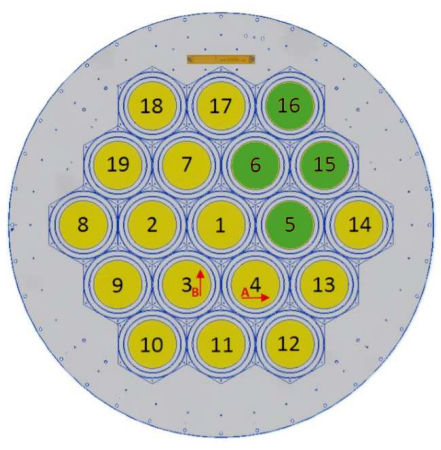

(b)

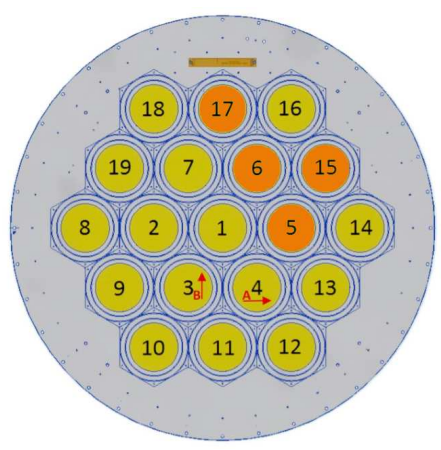

(c)

Figure 1: Schematics of muti-beam coincidence matching. a: A signal from the target (detected by beam 1) should not appear in the 6 outermost beams, otherwise it is RFI. b: An example of permitted signals in MBCM blind search. c: An example of forbidden signals in MBCM blind search. Beam 5 and beam 17 are separated by beam 6, thus an extra-terrestrial signal cannot cover both of them.

coincidence can be avoided. Moreover, examining the distribution of particular signals in 19 beams helps reveal the origin of instrumental RFI, since the patterns are related to the data transmission structure. Some deceptive instrumental RFI signals present characteristics similar to ETI signals, but are discernible by MBCM.

In this work, every observation lasts for 20 min (except for HD 111998, observed for 4 min). Our searches for ETI signals focus on narrow band $(\sim \mathrm{Hz})$ drifting signals, one of the most common signal types aimed for by radio $\mathrm{SET}^{4}-15 \mid 17$. Widely used in human electromagnetic communications, narrow band signals are indicative of technological sources because they cannot be produced by any natural astrophysical process, and can arise from either intentional transmission or leakage. A narrow band signal transmitted from a distant source drifts in frequency due to 
Doppler effect, and the drift rate is given by

$$
\dot{v}=\frac{v_{0}}{c} a,
$$

where $v_{0}$ is the emitted frequency from the transmitter, and $a$ is the relative acceleration between the transmitter and the receiver. Because the relative acceleration between the Earth and an exoplanet is unlikely to be exact zero, non-drift signals are determined as RFI directly, which originate from stationary ground-based interference sources and make up a considerable proportion of detected signals.

Some previous targeted SETI searched solely Stokes I data for narrow band drifting signals ${ }^{14 \mid 17}$. The data recorded by FAST spectral line backend consists of four polarizations, among which polarization 1 and 2 (pol1 and pol2) represent the intensity of two orthogonal directions ( $I_{\mathrm{X}}$ and $\left.I_{\mathrm{Y}}\right)$ respectively. We process the data of two orthogonal polarizations separately because we find that instrumental RFI usually appears much stronger in one polarization than another, such as harmonics produced by crystal oscillators, a major type of instrumental RFI. This imbalance is caused by the relative positions of the instrumental RFI sources and the data pipelines of two polarizations, and the different shielding levels of the pipelines to instrumental RFI. A signal that appears much stronger in one polarization than another is unlikely to come from an extra-terrestrial source. Even though ETI may emit linearly polarized signals, this phenomenon can hardly occur unless the polarization direction of the signal is almost aligned with one of the two polarization directions of the receiver during the observation and is not seriously affected by interstellar polarization.

Using turboSET ${ }^{29}$, a software package for targeted SETI, we search our data for narrow 
band drifting signals across a frequency range of $1.05-1.45 \mathrm{GHz}$ (FAST L-band) 18,2021 . The narrow band signals detected above the signal-to-noise ratio $(\mathrm{S} / \mathrm{N})$ threshold (taken as 10 in this work) are referred to as 'hits, $13,15,17$, and hits that detected only by the target beam (beam 1) but not by any of the reference beams (beam 8, 10, 12,14, 16 and 18) are referred to as 'events'. We find 1,309,503 hits from pol1 and 1,324,198 hits from pol2, among which we select 2,013 events from pol1 and 2,064 events from pol2, excluding $97.0 \%$ and $96.9 \%$ of the hits detected by beam 1 respectively.

The distributions of frequency, drift rate and $\mathrm{S} / \mathrm{N}$ for detected hits and events are shown in Fig 2 and 3. The results of RFI test at FAST site show two main types of RFI sources within the $1.05-1.45 \mathrm{GHz}$ frequency band: civil aviations and navigation satellites ${ }^{30}$. We find only a small fraction (4.2\% and 3.5\% for pol1 and pol2 respectively) of hits have frequencies within the civil aviation band. In contrast, hits within the navigation satellite band account for a considerable proportion (31.5\% and $31.6 \%$ for pol1 and pol2 respectively), implying they are possibly a major RFI source. The proportions of hits with positive, zero and negative drift rates are $5.6 \%, 65.8 \%$ and $28.6 \%$ for pol1, and $5.4 \%, 66.1 \%$ and $28.5 \%$ for pol 2 respectively. Non-drift hits are in the majority as expected since ground-based interference sources are mostly stationary. The bias towards negative drift rate results from the downward relative acceleration vectors of non-geosynchronous

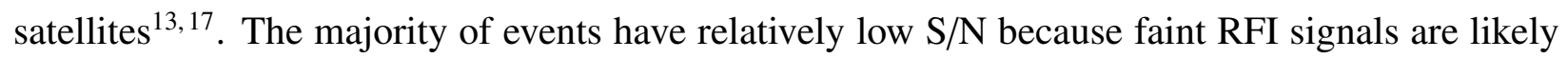
to fall short of the $\mathrm{S} / \mathrm{N}$ threshold thus escaping the detection by the reference beams.

Every event is re-examined by visual inspection of the dynamic time-frequency spectra (wa- 


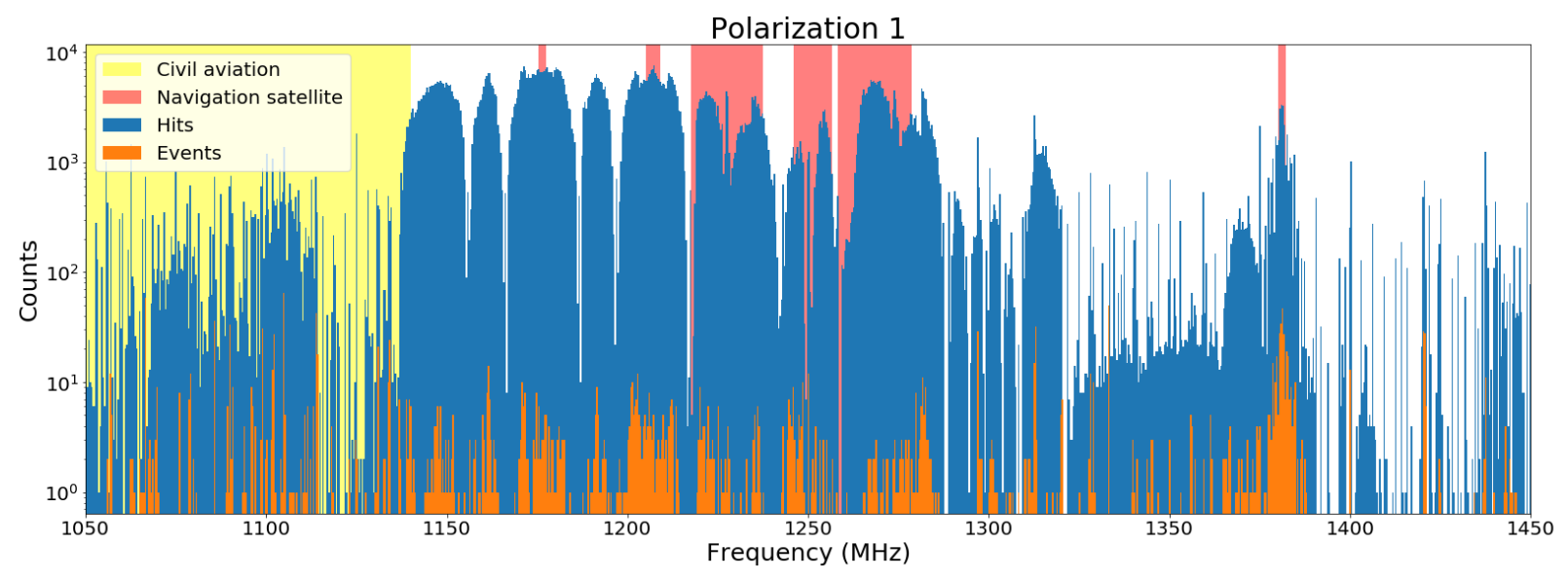

(a)

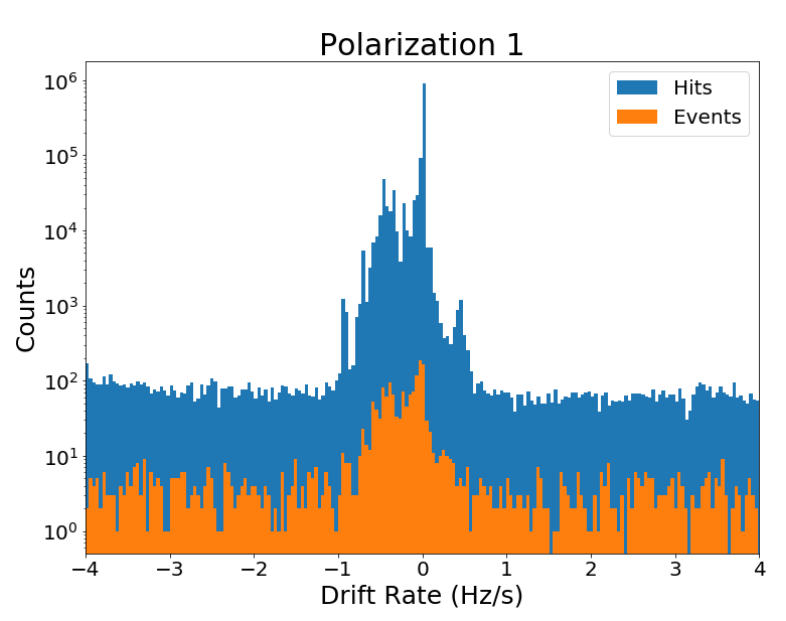

(b)

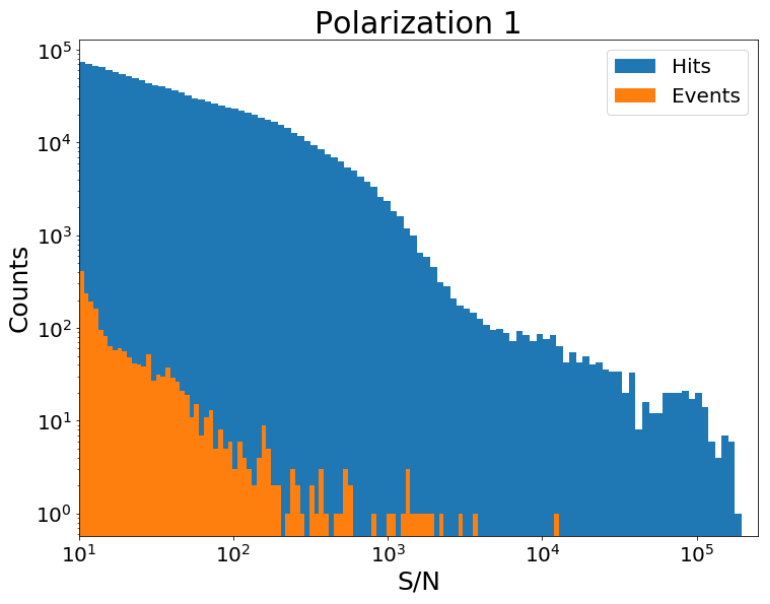

(c)

Figure 2: Histograms of hits and events for polarization 1 as functions of frequency, drift rate and S/N. Frequency bands of registered interference sources are displayed on the frequency panel. 


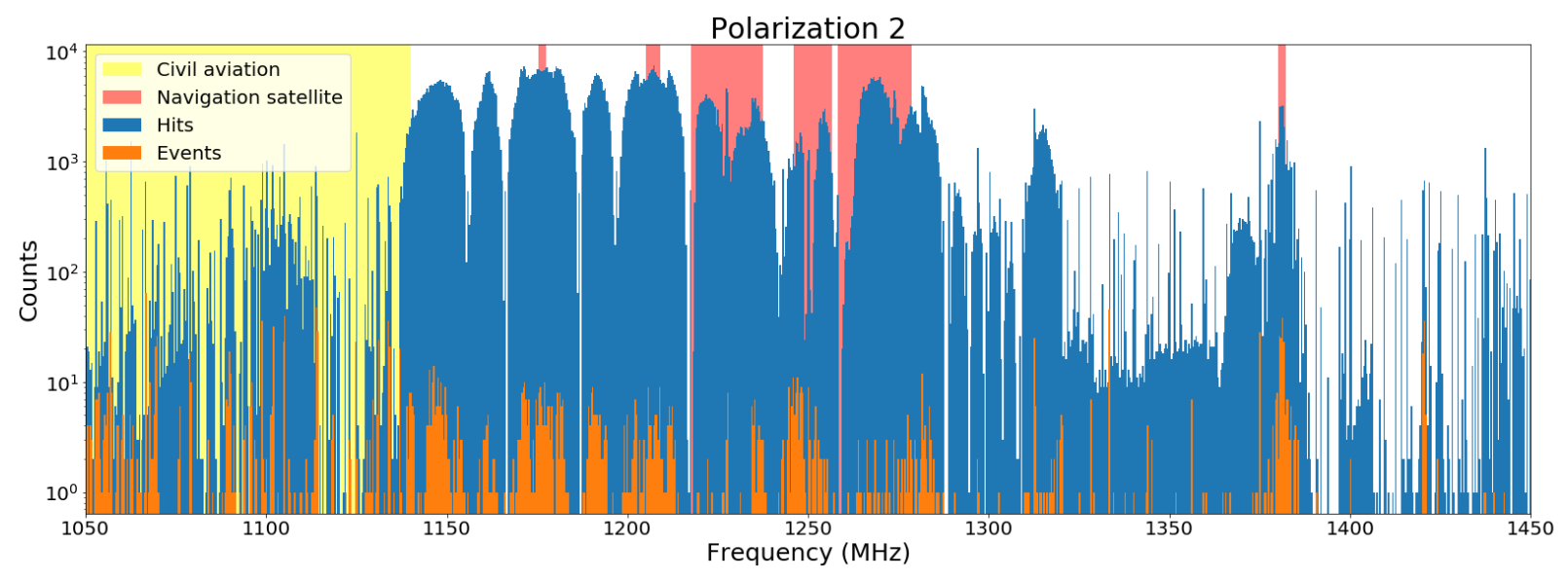

(a)

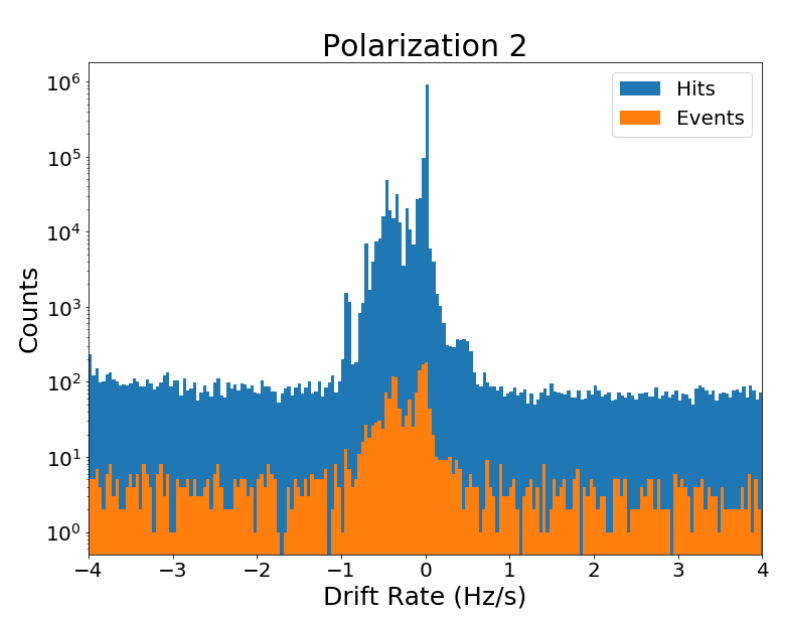

(b)

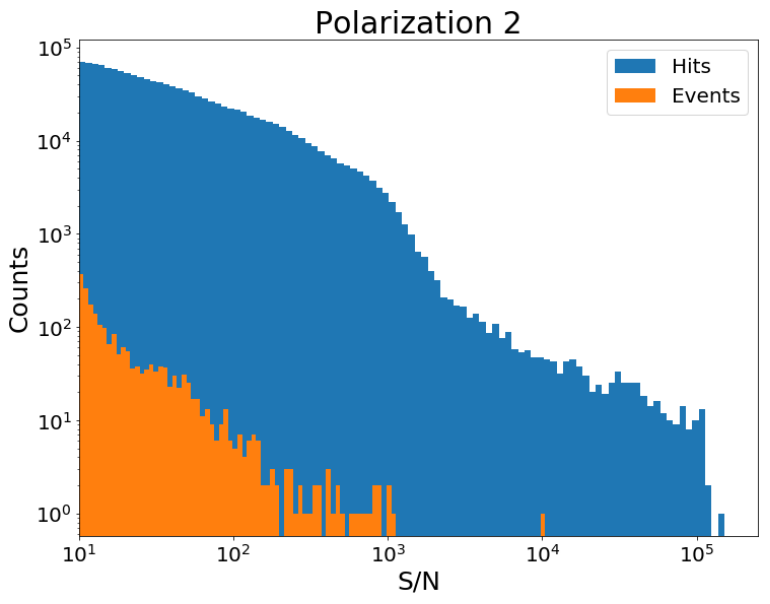

(c)

Figure 3: Histograms of hits and events for polarization 2 as functions of frequency, drift rate and $\mathbf{S} / \mathbf{N}$. Frequency bands of registered interference sources are displayed on the frequency panel. 
terfall plots) and detailed analysis of frequency and drift rate. All the events that have been confirmed as RFI can be classified into two categories. The first type of events are false positives, including two cases. One case is that there is no hit present on the waterfall plot of beam 1, but turboSETI detects a hit, while the other case is that there are hits present on the waterfall plots of some of the reference beams, but turboSETI fails to detect them. The second type of events are instrumental RFI resulting from inner interference sources, specifically, crystal oscillator harmonics.

A signal which peaked our interest is shown in Fig, 4, which is detected at $1140.604 \mathrm{MHz}$ from the observation towards Kepler-438, with a bandwidth of $\sim \mathrm{Hz}$ and a best-fit drift rate of -0.0678 , within the reach of a transmitter moving with an exoplanet. It is the only event that is only present in beam 1 and not in any other beam, which makes it different from any other event we detect. According to this feature and the fact that this event persists for 20 min, during which its drift rate varies slightly, we can exclude the possibility of all ground-based RFI sources outside the telescope, including airplanes. We also find that no satellite or deep-space probe entered the main lobe of beam 1 during the observation, thus the possibility of artificial objects is also ruled out.

Although some of its characteristics are somewhat consistent with a true ETI signal, there is still a piece of evidence leading us to suspect that the Kepler-438 event is an instrumental RFI signal. This event appears much stronger in pol2 than pol1, as shown in Fig 6. This is consistent with the aforementioned property of crystal oscillator harmonics. But its frequency has no relation with the fundamental frequencies of the crystal oscillators in the FAST instruments, and it only 
appears in beam 1, thus it is unlikely to be associated with crystal oscillator harmonics. So far, we are still uncertain about the exact origin and generation mechanism of the Kepler- 438 event, and more experimental re-observations are required. Even if this event is determined as instrumental RFI, the results will provide meaningful experience for RFI identification in future SETI.

The sensitivity of radio SETI observations can be measured by equivalent isotropic radiated power(EIRP) ${ }^{13] 14|17| 21}$, defined as

$$
\mathrm{EIRP}=4 \pi d^{2} \sigma \frac{2 k_{\mathrm{B}} T_{\mathrm{sys}}}{A_{\mathrm{eff}}} \sqrt{\frac{B}{n_{\mathrm{pol}} t_{\mathrm{obs}}}}
$$

where $d$ is the distance to the target, $\sigma$ is the $\mathrm{S} / \mathrm{N}$ threshold, $T_{\text {sys }}$ is the system temperature, $A_{\text {eff }}$ is the effective collecting area of the telescope, $B$ is the channel bandwidth, $n_{\mathrm{pol}}$ is the number of polarizations and $t_{\mathrm{obs}}$ is the observation time,respectively. The sensitivity $A_{\mathrm{eff}} / T_{\text {sys }}$ of FAST and its L-band 19-beam receiver is $\sim 2000 \mathrm{~m}^{2} / \mathrm{K}^{18-20}$. Considering our closest target, Ross 128 , of $3.37 \mathrm{pc}$ away ${ }^{31}$, we calculate EIRP $_{\min }=1.5 \times 10^{9} \mathrm{~W}$, achieving an unprecedented sensitivity. Comparing to the EIRP of the Arecibo Planetary Radar at $\sim 10^{13} \mathrm{~W}$, signals FAST can detect are well within the reach of current human technology.

As this application of multi-beam coincidence matching to targeted SETI, we verify its great advantages over traditional on-off strategy in both time efficiency and signal identification effectiveness by observing 33 sources using FAST and its L-band 19-beam receiver. In addition to FAST, MBCM can apply to any radio telescope with a multi-beam receiver, as long as the outermost beams are far enough away from the central beam to serve as reference beams. On the basis of MBCM, SETI will be further promoted when simultaneous observations by two or more 


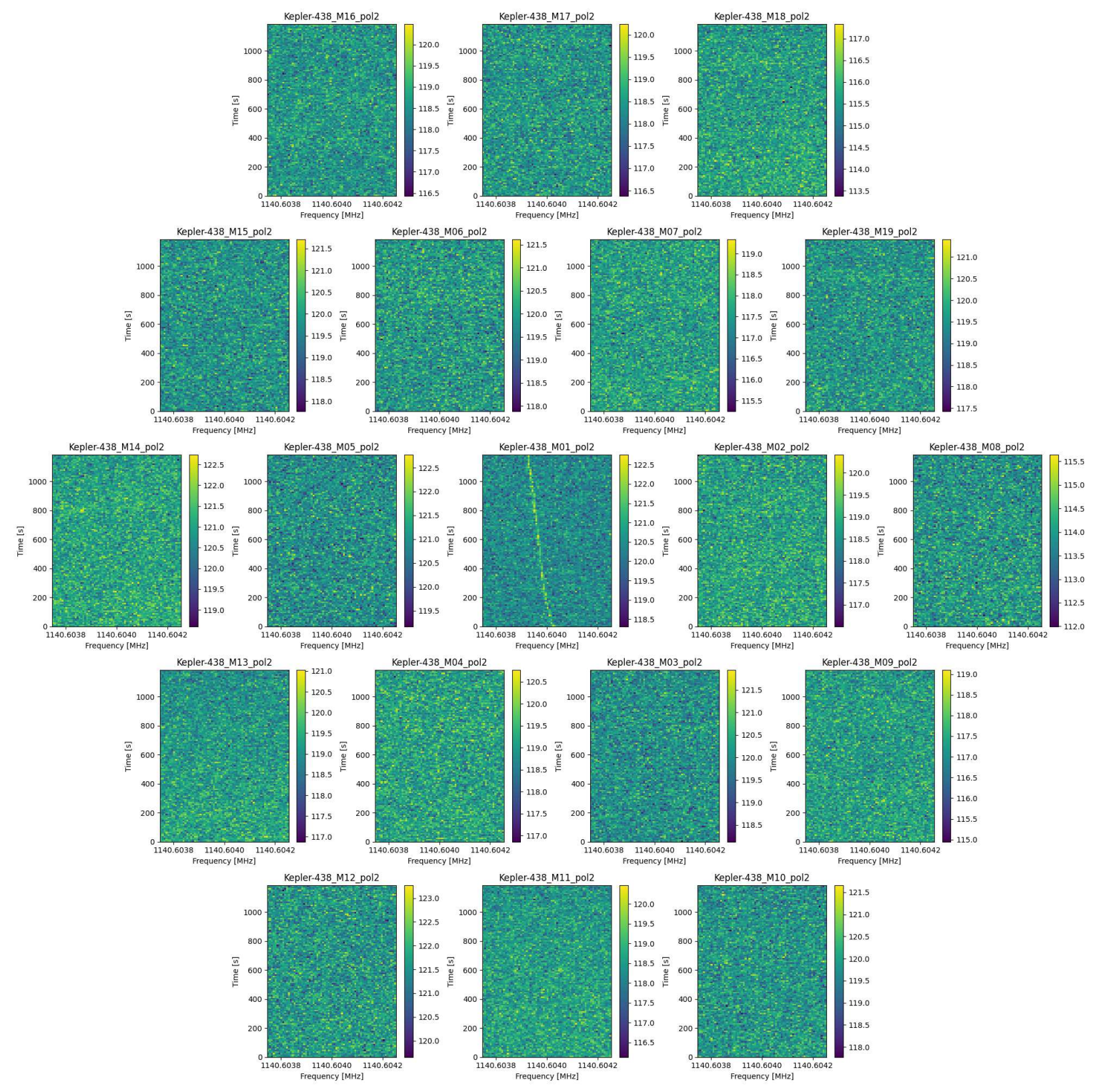

Figure 4: A signal at 1140.604 MHz detected during our 20-minute observation towards

Kepler-438. This signal appears only in beam 1, not in any other beam. 
telescopes become practicable ${ }^{28}$.

1. Seager, S. The future of spectroscopic life detection on exoplanets. Proceedings of the National Academy of Science 111, 12634-12640 (2014).

2. Tarter, J. The Search for Extraterrestrial Intelligence (SETI). Ann. Rev. Astron. Astrophys. 39, 511-548 (2001).

3. Zhang, B. A quantitative assessment of communicating extra-terrestrial intelligent civilizations in the galaxy and the case of FRB-like signals. Frontiers of Physics 15, 54502 (2020). 2006.08493.

4. Drake, F. D. Project Ozma. Physics Today 14, 40 (1961).

5. Verschuur, G. L. A Search for Narrow Band 21-cm Wavelength Signals from Ten Nearby Stars. 19, 329-340 (1973).

6. Tarter, J., Cuzzi, J., Black, D. \& Clark, T. A high-sensitivity search for extraterrestrial intelligence at $\gamma 18 \mathrm{~cm} . \mathbf{4 2}, 136-144$ (1980).

7. Valdes, F. \& Freitas, J., R. A. A search for the tritium hyperfine line from nearby stars. $\mathbf{6 5}$, $152-157$ (1986).

8. Horowitz, P. \& Sagan, C. Five Years of Project META: an All-Sky Narrow-Band Radio Search for Extraterrestrial Signals. Astrophys. J. 415, 218 (1993). 
9. Backus, P. R. \& Project Phoenix Team. Project Phoenix: SETI Observations from 1200 to $1750 \mathrm{MHz}$ with the Upgraded Arecibo Telescope. In Stanimirovic, S., Altschuler, D., Goldsmith, P. \& Salter, C. (eds.) Single-Dish Radio Astronomy: Techniques and Applications, vol. 278 of Astronomical Society of the Pacific Conference Series, 525-527 (2002).

10. Siemion, A. P. V. et al. A 1.1-1.9 GHz SETI Survey of the Kepler Field. I. A Search for Narrow-band Emission from Select Targets. Astrophys. J. 767, 94 (2013). 1302.0845.

11. Harp, G. R. et al. SETI Observations of Exoplanets with the Allen Telescope Array. Astron. J. 152, 181 (2016). 1607.04207.

12. Gray, R. H. \& Mooley, K. A VLA Search for Radio Signals from M31 and M33. Astron. J. 153, 110 (2017). 1702.03301.

13. Enriquez, J. E. et al. The breakthrough listen search for intelligent life: 1.1-1.9 GHz observations of 692 nearby stars. The Astrophysical Journal 849, 104 (2017). URL https: //doi.org/10.3847/1538-4357/aa8d1b.

14. Price, D. C. et al. The breakthrough listen search for intelligent life: Observations of 1327 nearby stars over 1.10-3.45 GHz. The Astronomical Journal 159, 86 (2020). URL https: //doi.org/10.3847/1538-3881/ab65f1.

15. Traas, R. et al. The Breakthrough Listen Search for Intelligent Life: Searching for Technosignatures in Observations of TESS Targets of Interest. Astron. J. 161, 286 (2021). 2101.11137.

16. Gajjar, V. et al. The Breakthrough Listen Search For Intelligent Life Near the Galactic Center. I. Astron. J. 162, 33 (2021). 2104.14148. 
17. Smith, S. et al. A radio technosignature search towards Proxima Centauri resulting in a signal of interest. Nature Astronomy (2021).

18. Nan, R. et al. The Five-Hundred Aperture Spherical Radio Telescope (fast) Project. International Journal of Modern Physics D 20, 989-1024 (2011). 1105 . 3794.

19. Li, D. \& Pan, Z. The Five-hundred-meter Aperture Spherical Radio Telescope Project. Radio Science 51, 1060-1064 (2016). 1612 .09372.

20. Jiang, P. et al. Commissioning progress of the FAST. Science China Physics, Mechanics, and Astronomy 62, 959502 (2019). 1903.06324.

21. Li, D. et al. Opportunities to search for extraterrestrial intelligence with the FAST. Research in Astronomy and Astrophysics 20, 078 (2020).

22. Nan, R. Five hundred meter aperture spherical radio telescope (FAST). Science in China: Physics, Mechanics and Astronomy 49, 129-148 (2006).

23. Zhang, Z.-S. et al. First SETI Observations with China's Five-hundred-meter Aperture Spherical Radio Telescope (FAST). Astrophys. J. 891, 174 (2020). 2002.02130.

24. Kasting, J. F., Whitmire, D. P. \& Reynolds, R. T. Habitable Zones around Main Sequence Stars. 101, 108-128 (1993).

25. Williams, D. M. \& Pollard, D. Earth-like worlds on eccentric orbits: excursions beyond the habitable zone. International Journal of Astrobiology 1, 61-69 (2002). 
26. Kopparapu, R. K. et al. Habitable Zones around Main-sequence Stars: New Estimates. Astrophys. J. 765, 131 (2013). 1301.6674.

27. Kaltenegger, L. \& Faherty, J. K. Past, present and future stars that can see Earth as a transiting exoplanet. Nature 594, 505-507 (2021).

28. Sheikh, S. Z. et al. Analysis of the Breakthrough Listen signal of interest blc1 with a technosignature verification framework. Nature Astronomy (2021).

29. Enriquez, E. \& Price, D. turboSETI: Python-based SETI search algorithm (2019). 1906.006.

30. Wang, Y. et al. Satellite RFI mitigation on FAST. Research in Astronomy and Astrophysics 21, $018(2021)$.

31. Gaia Collaboration et al. Gaia Early Data Release 3. Summary of the contents and survey properties. Astron. E Astrophys. 649, A1 (2021). 2012.01533.

32. Price, D., Enriquez, J., Chen, Y. \& Siebert, M. Blimpy: Breakthrough Listen I/O Methods for Python. The Journal of Open Source Software 4, 1554 (2019).

33. Torres, G. et al. Validation of 12 Small Kepler Transiting Planets in the Habitable Zone. Astrophys. J. 800, 99 (2015). 1501.01101.

Acknowledgements This work was supported by the National Science Foundation of China (NSFC) Programs Grants No. 11929301 and National Key RD Program of China (2017YFA0402600), the National Natural Science Foundation of China Grants No. 12041304, CAS “Light of West Chin” Program, Cultivation Project for FAST Scientific Payoff and Research Achievement of CAMS-CAS. This work made use of 
data from FAST, a Chinese national mega-science facility built and operated by the National Astronomical Observatories, Chinese Academy of Sciences.

Author Contributions T.J.Z., D.W. and V. G. launched the FAST observational campaign on PT20200169 in the FAST free application; Z.Z.T.and H.C.Z coordinated the FAST observations; FAST observations, instrument setting and monitoring was done by Z.Z.T. Y.Z. adapted the backend of FAST spectrum line to generate high frequency resolution spectral products, and provided important backend RFI information; H.Y.Z. provided FAST satellite RFI database, and contributed to the scientific analysis; T.J.Z. Z.Z.T., H.C.Z. drafted the paper; Z.Z.T., H.C.Z., Z.S.Z. and V. G. processed the data; All authors contributed to the discussion, analysis and interpretation of the data and to the final version of the manuscript.

Competing Interests The authors declare that they have no competing financial interests.

\section{Methods}

Data processing We record our data on the spectral line backend with L-band 19-beam receiver across the $1.0-1.5 \mathrm{GHz}$ bandwidth. Because the effective bandwidth of the receiver is $1.05-$ $1.45 \mathrm{GHz} \frac{18}{2021}$, we discard the events detected within both ends of the band after searching by turboSETI. Our data have a frequency resolution of $\sim 7.5 \mathrm{~Hz}$ and the integration time of each spectrum is 10 seconds. Each FITS file contains two spectra recorded by one beam, and the total volume of our data is 66.5 TB (including experimental observations). The FITS files of each beam and each target are combined and converted into two filterbank files (pol1 and pol2), a data format accessible to the Blimpy Python package ${ }^{32}$. 
TurboSETI ${ }^{1329}$ is a Python/Cython package using the tree search algorithm ${ }^{10}$ to search for narrow band signals with arbitrarily large drift rates, breaking the limitation of time-frequency resolution by shifting arrays. Two essential parameters required by turboSETI are an $\mathrm{S} / \mathrm{N}$ threshold and a maximum drift rate (MDR), allowing turboSETI to search for narrow band signals above the

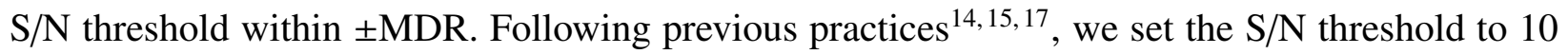
and the MDR to $4 \mathrm{~Hz} / \mathrm{s}$.

We use the find_event_pipeline of turboSETI to select events and reject obvious RFI. The RFI identification criteria in the original code applies to on-off strategy, where on- and off- observations are taken in sequence, thus the same RFI signal appears at different frequencies in a series of observations due to Doppler drift. Because MBCM observes a target and reference locations simultaneously, an RFI signal is expected to appear at the same frequency in all beams. We modify the code and set the RFI rejection range as $\pm 3 \delta v$, where $\delta v$ is our frequency resolution. That is, if a hit present in beam 1 is accompanied by any hit within this range in the reference beams, the hit will be determined as RFI.

Signal identification As mentioned above, we can rank SETI signal from low to high level as: hit, event, candidate and signal of interest or ETI signal. Events passing the find_event_pipeline of turboSETI are not certainly real candidate signals, and every event requires examination from many aspects. 


\subsubsection{Visual inspection}

Most events (90 - 95\% in this work) are apparent false positives and can be excluded by visual inspection of the waterfall plots $\left.{ }^{13}+15 \mid 17\right] 28$. Firstly, only the plots of beam 1 and the 6 reference beams are required. The false positives display either invisible but detected hits in beam 1 or visible but undetected hits in the reference beams on the plots. The latter are mainly caused by the hits in the reference beams below the $\mathrm{S} / \mathrm{N}$ threshold. After removing these false positives, we then make the plots of all 19 beams for the remaining events to examine their beam coverage. This procedure is to determine whether they satisfy the criteria mentioned above: an extra-terrestrial signal cannot cover two beams that are separated by one or more beams.

\subsubsection{Harmonic analysis}

Comparing the events passing visual inspection with each other, we find the frequencies of nearly all these events are concentrated on several specific values, which are added by integer multiples of the fundamental frequencies $(33.333 \mathrm{MHz}$ and $125 \mathrm{MHz}$ ) of the crystal oscillators in the FAST instruments. These events are attributed to harmonics generated by the crystal oscillators. The frequency values are not that precise because the frequencies of the crystal oscillators are unstable and subject to various factors like temperature. This makes the drift rates of the harmonics vary rapidly, giving them a curved morphology on the waterfall plots. Harmonics generated from the same source at the same time have identical morphology, though their drift directions can be mirrored. Because the data of every two beams are analyzed by the same field programmable gate 
array (FPGA) except for beam 1923, signals generated by crystal oscillators always appear in two adjacent beams simultaneously. Most harmonics appear much stronger in one polarization than another.

In this work, we can exclude all the events selected by turboSETI except the Kepler-438 event through the above procedures. The following procedures are used to further examine this particular event and applicable to various events that cannot be excluded by the above procedures.

\subsubsection{Artificial objects}

Though MBCM excludes various ground-based RFI directly, signals transmitted by satellites and deep-space probes are still likely to confound our identification. Due to the short orbital periods ( 90 min) of low-Earth orbit satellites, their signals are impossible to cover a long time in the data $^{28}$. As they move across the sky, their signals must also be detected by beams other than beam 1, thus unlikely to be selected as events by MBCM. However, medium-Earth and geosynchronous orbit satellites are still potential RFI sources. The trajectories of satellites passing overhead during the observation can be queried by FAST satellite RFI database ${ }^{30}$, where we find no satellite that entered the region within 3 degrees of the main lobe of beam 1 as we observed Kepler-438. To determine whether the Kepler-438 event is a satellite signal captured by the side lobes, we can calculate the drift rates caused by the relative accelerations of the satellites and compare them with the Kepler-438 event. But this possibility is very low, especially for a signal that persists for 20 min with only a slight change in the drift rate. Deep-space probes are the most stationary artificial 
objects in the sky. Due to their remote distances, their signals are too weak to be detected by the side lobes. They are only detectable when they happen to be in or near the main lobe of beam 1. The coordinates of all deep-space probes during the observation can be queried from NASA Horizons (https://ssd.jpl.nasa.gov/horizons.cgi). We find no probe that overlapped with Kepler438.

\subsubsection{Homologous RFI excision}

Signals generated from the same source share similar characteristics or have regular patterns in frequency, drift rate and morphology, so a signal sharing the same origin with some RFI signals can be determined as $\mathrm{RF}^{28}$. Some transmitters can emit signals at several different frequencies simultaneously, and some can always emit signals at some constant frequencies. Therefore, there are two directions in which we can search for homologous RFI for a particular signal: RFI with the same frequency-normalized drift rate $\dot{v} / v$ from data recorded at the same time and RFI at the same frequency in observations towards other sources.

For the former case, the morphology of homologous RFI signals should be similar, and their beam coverage should be the same. The frequency spacings between them are integer multiples of a fundamental frequency if they are produced by intermodulation. From the data of the observation towards Kepler-438, we find some RFI signals with $\dot{v} / v$ similar to the Kepler-438 event. However, none of them has the same morphology with the Kepler-438 event.

For the latter case, the beam coverage should be compared between the particular event and 
possible homologous RFI to determine whether they share the same origin. From observations towards other 8 sources, we find 8 weak drifting signals in the range of $1140.604 \mathrm{MHz} \pm 2.5 \mathrm{kHz}$. All the 8 signals appear only in beam 1 and pol2, but fail to reach the $\mathrm{S} / \mathrm{N}$ threshold (Fig.7). However, the frequencies of these 8 signals are all $\sim 200 \mathrm{~Hz}$ lower than $1140.604 \mathrm{MHz}$ and reveal coherent drift behaviours, while the Kepler-438 event looks out of place with them. Additionally, these 8 sources are observed between April 19 and 21, while Kepler-438 is the only source observed on June 29. Hence, we infer that these 8 signals are correlated and generated by the same RFI source, but the Kepler-438 event is unlikely to come from the same source as that of these 8 signals.

\subsubsection{Re-observation}

Using MBCM strategy, we perform a re-observation towards Kepler-438 on Novermber 7, which also lasts for $20 \mathrm{~min}$. By visually inspecting the waterfall plots, we find no signal at $1140.604 \mathrm{MHz}$ $\pm 2.5 \mathrm{kHz}$ again.

\subsubsection{Candidate signal verification framework}

Our trial to identify the Kepler-438 event constructs a candidate signal verification framework applicable to MBCM targeted SETI observations, not only for FAST, but also for any telescope with a multi-beam receiver:

1. Verify that the event is not a false positive by visual inspection of the waterfall plots. A false positive has either invisible signal in the target beam or visible signal in the reference beams. 
2. Verify that the beam coverage conforms to the characteristics of an extra-terrestrial signal, not covering two beams that are separated by one or more beams.

3. Verify that the event is not a crystal oscillator harmonic. A harmonic has a frequency added by integer multiples of the fundamental frequencies and appears simultaneously in beams connected to the same FPGA.

4. Verify that the event is present in both polarizations, rather than in only one polarization, and the difference between the intensities of two polarizations is not too great.

5. Verify that the frequency of the event is not within the registered RFI bands at the observatory site. If not, compare the drift rate to the motion of the corresponding sources.

6. Query the positions of satellites and deep-space probes during the observation and determine whether they can affect the observation. If so, compare the drift rate to the motion of the corresponding artificial objects.

7. Search for RFI signals with the same $\dot{v} / v$ and morphology from the same observation. If any, look for regularity in the frequency spacings among them to determine whether they are produced by intermodulation.

8. Search for RFI signals at the same frequency from observations towards other sources by the same telescope. If any, compare their beam coverage to determine whether they have the same source.

This framework assumes that all instrumentation of the telescope is functioning properly and 
that there is no serious disturbance to the observations. We list harmonic analysis as the third step because in our work crystal oscillator harmonics account for most of the signals passing the first two steps. For instruments better shielded from crystal oscillator harmonics, this step can be done later. If a signal passes all of the above verification steps, the source from which the signal is detected can be re-observed in order to re-detect the signal, which can increase the credibility of the candidate signal and make it a signal of interest $\sqrt{28}$. Even though the signal fails to be re-detected, it is still a good candidate signal.

Drift rate analysis Though turboSETI provides a first-order best-fit drift rate by the tree search algorithm for every hit, we can determine the drift rate to higher precision by a de-drifting algorithm to further analyze the characteristics of particular signals 28 .

According to the first-order drift rate estimated by turboSETI, we set a drift rate test range and step size for a specific signal. Then we intercept a time-frequency-power array around the frequency of the signal. At each drift rate, every time row in the time-frequency-power array is shifted by the corresponding number of pixels, calculated by

$$
\text { number of pixels }=\left[\text { row index } \times \text { drift rate } \times \frac{\delta t}{\delta v}\right] \text {, }
$$

to form a stair-like array, and this procedure is named 'de-drift'. Once the array is de-drifted, the spectra in the array are summed over the observation time. From this one-dimensional spectrum, we search for a power maximum in frequency space, and each drift rate corresponds to such a maximum. The drift rate that yields the largest power maximum is the best-fit one. The precision of the drift rate obtained by this algorithm is higher than that fitted by the tree search algorithm. 
The relative acceleration resulting in the Doppler drift of extra-terrestrial signals is mainly caused by the rotation and orbit of the Earth and the transmitter. The drift rate caused by the Earth's rotation at FAST's latitude when $v_{0}=1.45 \mathrm{GHz}$ is

$$
\dot{v}_{\oplus, \mathrm{r}}=\frac{v_{0}}{c} \frac{4 \pi^{2} R_{\oplus}}{P_{\oplus}^{2}} \cos \phi=0.15 \mathrm{~Hz} / \mathrm{s},
$$

and that caused by the Earth's orbit is

$$
\dot{v}_{\oplus, \mathrm{o}}=\frac{v_{0}}{c} \frac{G M_{\odot}}{r_{\oplus}^{2}}=0.03 \mathrm{~Hz} / \mathrm{s} .
$$

For a transmitter located on a planet in the habitable zone around an M-type star, such as Kepler$438 \mathrm{~b}$, the drift rate caused by the planetary orbit is

$$
\dot{v}_{\mathrm{p}, \mathrm{o}}=\frac{v_{0}}{c} \frac{G M_{\star}}{r_{\mathrm{p}}^{2}},
$$

yielding $0.57 \mathrm{~Hz} / \mathrm{s}$ for Kepler- $438 \mathrm{~b}^{33}$. Though it is hard to measure the rotational period of an exoplanet, such planets are expected to have been tidally locked. The calculation of exact relative acceleration is complicated and requires detailed information of orbital elements and rotational parameters. Actually, such calculation is not necessary, because even if the drift rate of a candidate signal is out of the range derived from celestial mechanics, this will not invalidate the signal because we do not know how the transmitter moves and whether the signal drifts electronically. In other words, drift rate analyses can give support to a candidate signal, but cannot be the only evidence to reject a candidate signal. Anyway, the above calculations are sufficient to verify that the drift rate of the Kepler- 438 event, $-0.0678 \mathrm{~Hz} / \mathrm{s}$, is celestial-mechanically attainable, and that the MDR we set for turboSETI, $4 \mathrm{~Hz} / \mathrm{s}$, is large enough to search for signals transmitted from a typical planet in habitable zone. 


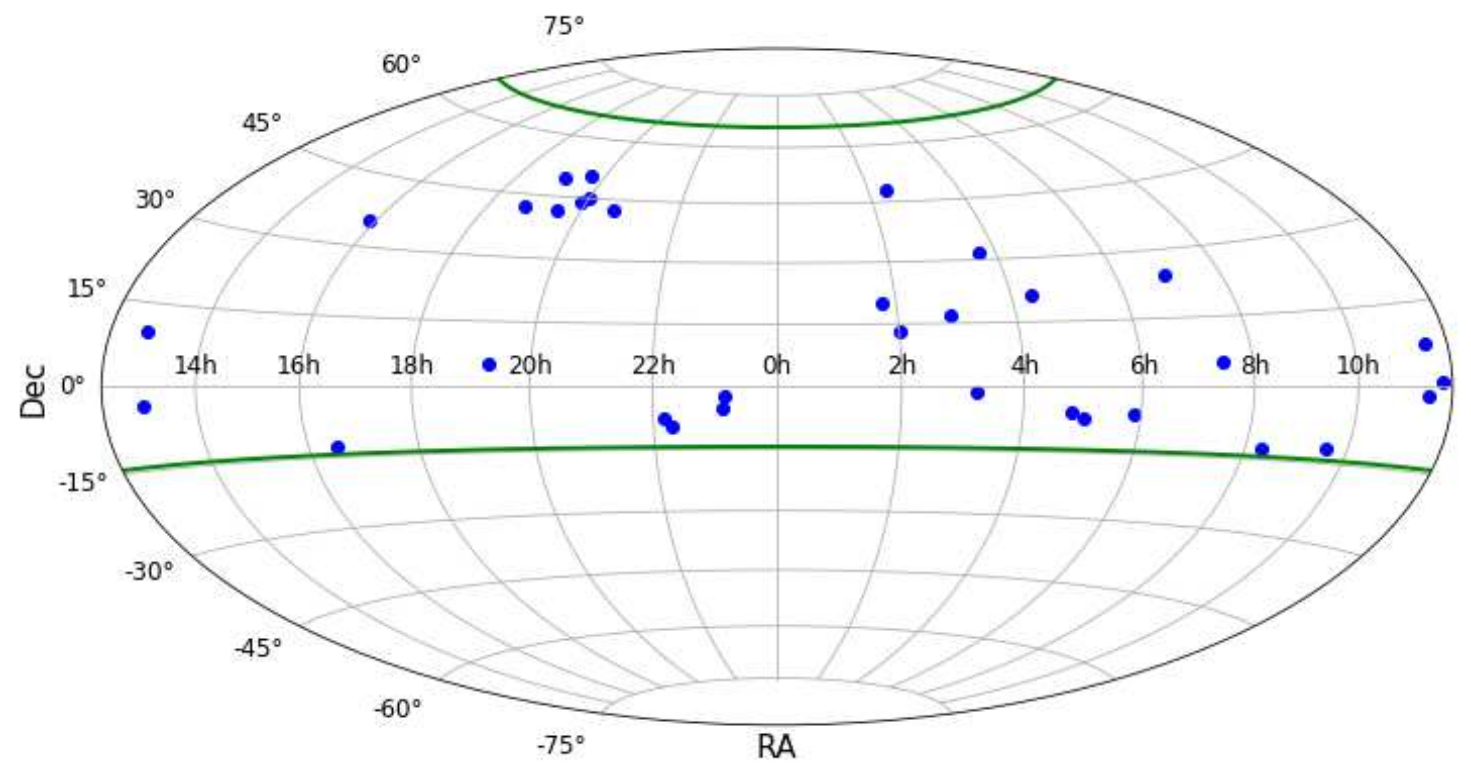

(a)

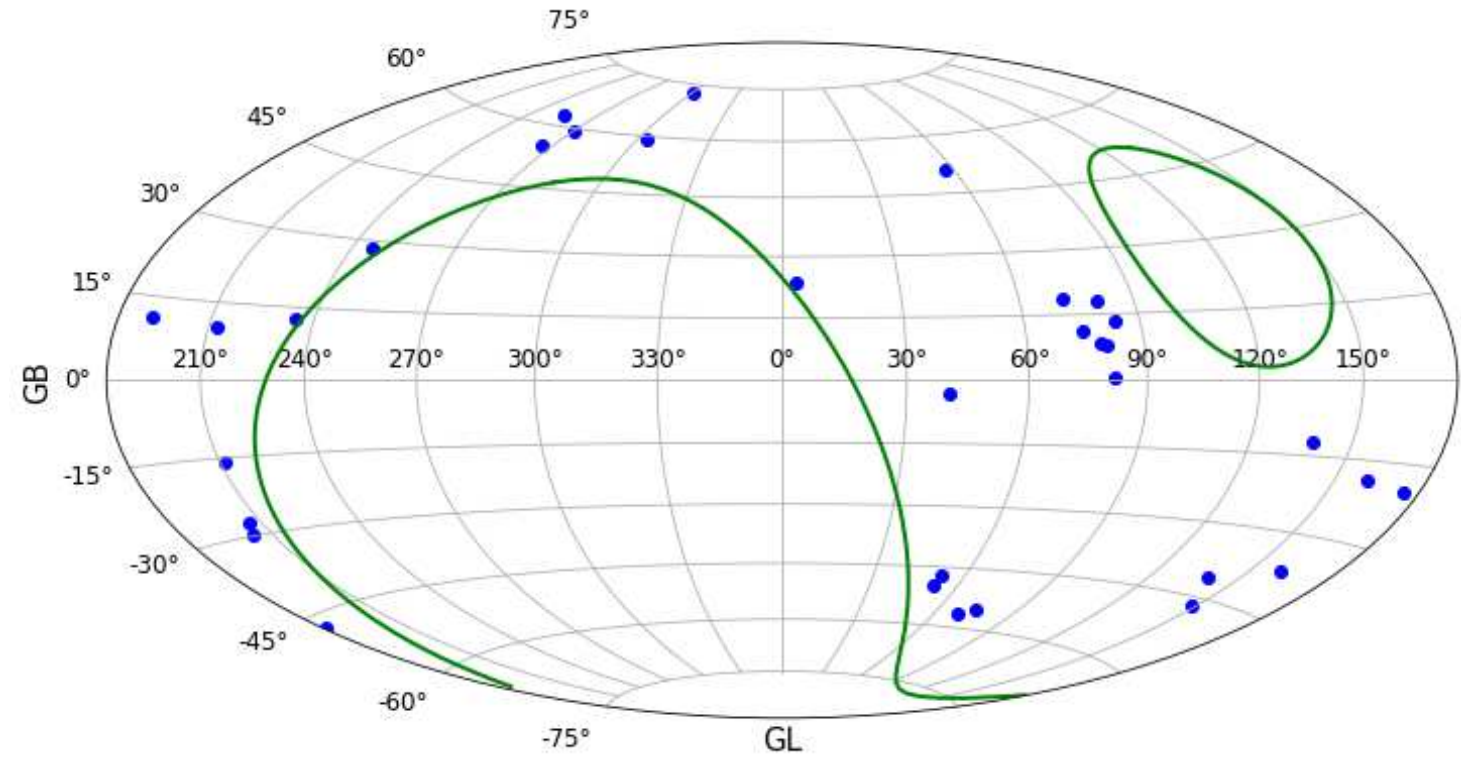

(b)

Figure 5: Distribution of 33 observed targets in equatorial and galactic coordinates. Limits of the observable sky of FAST are depicted by green curves. 

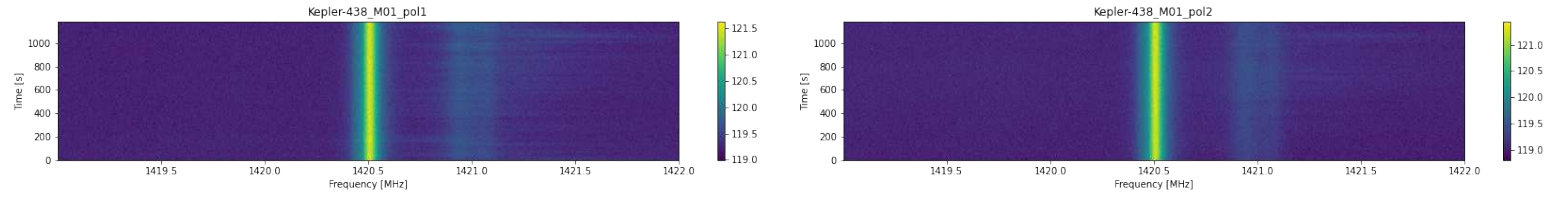

(a)
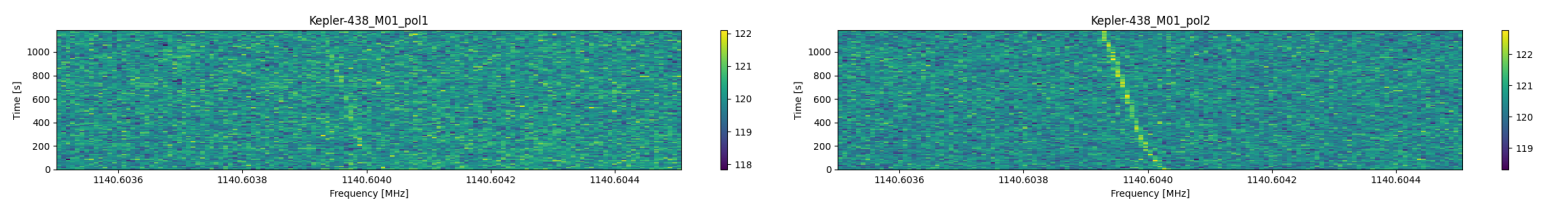

(b)

Figure 6: Polarized waterfall plots of the neutral hydrogen line (top panel) and the event detected at 1140.604 MHz (bottom panel) from the observation towards Kepler-438. The left panels show the plots of pol1 and the right panels show the plots of pol2. The intensities of the neutral hydrogen line in two polarizations are almost the same, while the Kepler-438 event appears much stronger in pol2 than pol1 suggesting that it is likely to be instrumental RFI. 

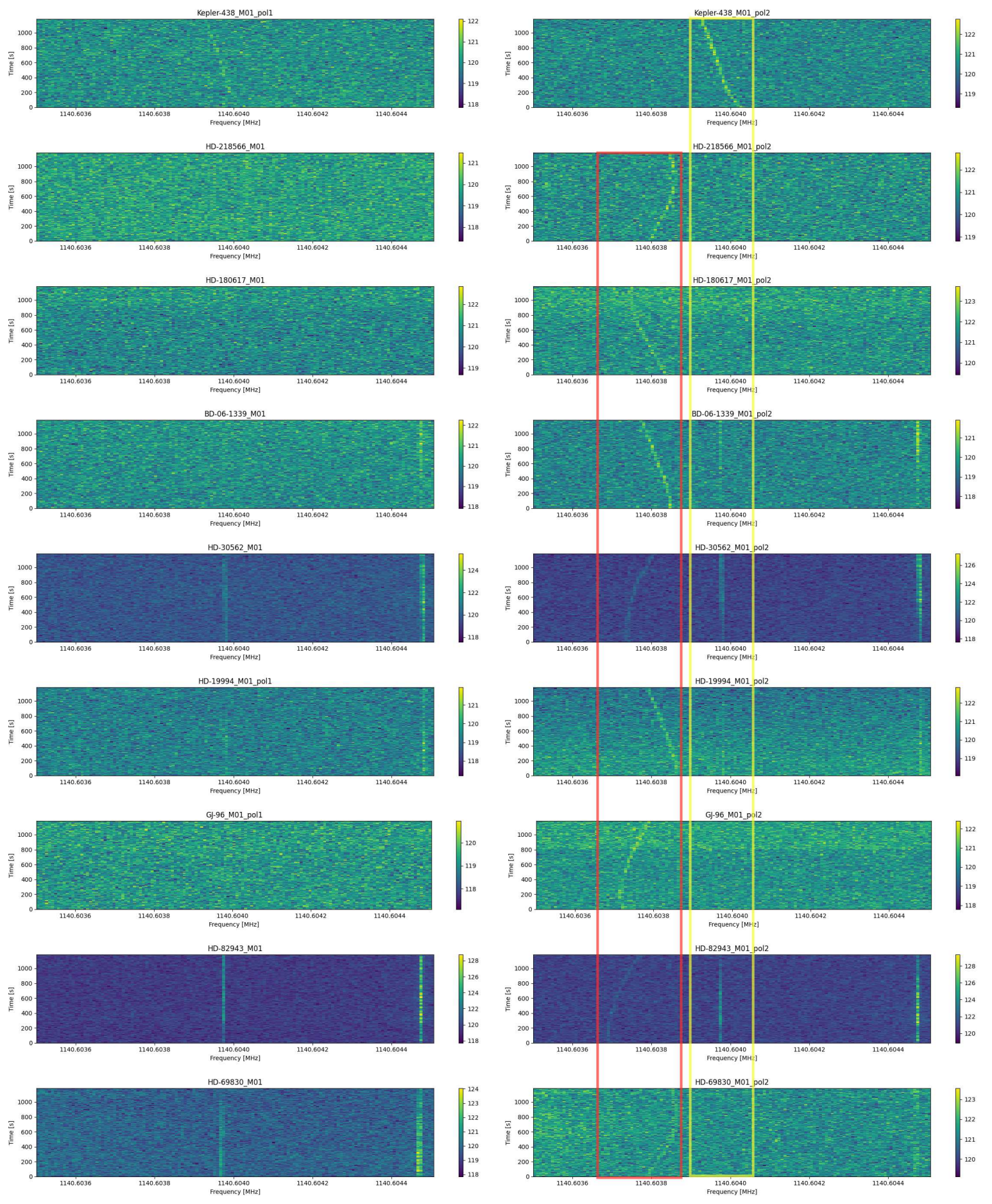

Figure 7: Polarized waterfall plots around 1140.604 MHz of the observations towards Kepler-

438 and other 8 sources. For simplicity, only the plots of beam 1 are displayed. The left panels show the plots of pol1 and the right panels show the plots of pol2. The frequencies of the latter 8 28 signals (red lines) are lower than that of the first signal (yellow lines). 
Table 1: Information of targets and observations

\begin{tabular}{|c|c|c|c|c|c|c|}
\hline Star Name & Gaia EDR3 ID & Epoch & RA & $\overline{\text { Dec }}$ & Distance (pc) & Observation Date \\
\hline HD 69830 & 5726982995343100928 & 2016 & $08: 18: 24.25$ & $-12: 38: 11.6$ & 12.58 & $2021-04-19$ \\
\hline HD 82943 & 5691782130578684544 & 2016 & $09: 34: 50.74$ & $-12: 07: 49.2$ & 27.69 & 2021-04-19 \\
\hline GJ 96 & 354077348697687424 & 2016 & $02: 22: 14.99$ & $+47: 52: 48.8$ & 11.95 & 2021-04-20 \\
\hline HD 19994 & 3265335443260522112 & 2016 & $03: 12: 46.64$ & $-01: 11: 47.1$ & 22.88 & 2021-04-20 \\
\hline HD 30562 & 3188395880157173120 & 2016 & $04: 48: 36.72$ & $-05: 40: 30.5$ & 26.14 & $2021-04-20$ \\
\hline BD-06 1339 & 3022099969137163904 & 2016 & $05: 53: 00.28$ & $-05: 59: 47.0$ & 20.31 & $2021-04-20$ \\
\hline HD 180617 & 4293318823182081408 & 2016 & $19: 16: 54.64$ & $+05: 09: 46.7$ & 5.92 & 2021-04-21 \\
\hline HD 218566 & 2638410646295370880 & 2016 & $23: 09: 11.40$ & $-02: 15: 40.2$ & 28.82 & 2021-04-21 \\
\hline Teegarden's Star & 35227046884571776 & 2016 & $02: 53: 04.71$ & $+16: 51: 51.7$ & 3.83 & $2021-05-25$ \\
\hline GJ 3323 & 3187115498866675456 & 2016 & $05: 01: 56.83$ & $-06: 56: 54.9$ & 5.37 & $2021-05-25$ \\
\hline GJ 273 & 3139847906307949696 & 2016 & $07: 27: 25.11$ & $+05: 12: 33.8$ & 3.79 & $2021-05-25$ \\
\hline 16 Cyg B & 2135550755683407232 & 2016 & $19: 41: 51.75$ & $+50: 31: 00.5$ & 21.13 & $2021-05-26$ \\
\hline HD 210277 & 2619706544757416192 & 2016 & $22: 09: 29.96$ & $-07: 33: 02.4$ & 21.34 & $2021-05-26$ \\
\hline TRAPPIST- 1 & 2635476908753563008 & 2016 & $23: 06: 30.37$ & $-05: 02: 36.7$ & 12.47 & $2021-05-26$ \\
\hline Wolf 1061 & 4330690742322011520 & 2016 & $16: 30: 17.96$ & $-12: 40: 04.3$ & 4.31 & $2021-06-06$ \\
\hline $\mathrm{K} 2-72$ & 2615653023342995584 & 2016 & $22: 18: 29.47$ & $-09: 36: 43.2$ & 66.51 & 2021-06-21 \\
\hline HD 10697 & 95652018353917056 & 2016 & $01: 44: 55.78$ & $+20: 04: 57.7$ & 33.16 & $2021-06-21$ \\
\hline HD 22781 & 217334764042444288 & 2016 & $03: 40: 49.58$ & $+31: 49: 33.1$ & 32.56 & $2021-06-21$ \\
\hline HD 50554 & 3380479015342121600 & 2016 & $06: 54: 42.78$ & $+24: 14: 42.5$ & 31.07 & 2021-06-21 \\
\hline $\mathrm{K} 2-3$ & 3796690380302214272 & 2016 & $11: 29: 20.49$ & $-01: 27: 18.5$ & 43.98 & 2021-06-21 \\
\hline HD 111998 & 3679242575447826432 & 2016 & $12: 53: 10.88$ & $-03: 33: 11.2$ & 33.44 & 2021-06-21 \\
\hline kap CrB & 1372702716380418688 & 2016 & $15: 51: 13.92$ & $+35: 39: 21.0$ & 29.99 & $2021-06-21$ \\
\hline Kepler-1649 & 2125699062780742016 & 2016 & $19: 30: 00.71$ & $+41: 49: 47.9$ & 92.76 & $2021-06-22$ \\
\hline Kepler-186 & 2079000330051813504 & 2016 & $19: 54: 36.66$ & $+43: 57: 18.0$ & 177.51 & $2021-06-22$ \\
\hline Kepler-560 & 2082162147537254400 & 2016 & $20: 00: 49.60$ & $+45: 01: 05.7$ & 109.43 & $2021-06-22$ \\
\hline HD 197037 & 2066437688140905600 & 2016 & $20: 39: 32.87$ & $+42: 14: 51.2$ & 33.08 & $2021-06-22$ \\
\hline Kepler-296 & 2132069633148965888 & 2016 & 19:06:09.61 & $+49: 26: 14.1$ & 219.60 & $2021-06-22$ \\
\hline Kepler-438 & 2104675781979819776 & 2016 & $18: 46: 34.97$ & $+41: 57: 03.8$ & 179.88 & $2021-06-29$ \\
\hline GJ 9066 & 76868614540049408 & 2016 & $02: 00: 14.16$ & $+13: 02: 38.7$ & 4.47 & $2021-09-10$ \\
\hline $\mathrm{K} 2-155$ & 145333927996558976 & 2016 & $04: 21: 52.71$ & $+21: 21: 11.7$ & 72.93 & $2021-09-10$ \\
\hline $\mathrm{K} 2-18$ & 3910747531814692736 & 2016 & $11: 30: 14.43$ & $+07: 35: 16.1$ & 38.10 & 2021-09-10 \\
\hline Ross 128 & 3796072592206250624 & 2016 & $11: 47: 45.05$ & $+00: 47: 56.8$ & 3.37 & $2021-09-10$ \\
\hline Gliese 486 & 3735000631158990976 & 2016 & $12: 47: 55.53$ & $+09: 44: 57.7$ & 8.08 & 2021-09-21 \\
\hline
\end{tabular}

Table 2: Events that pass visual inspection

\begin{tabular}{|c|c|}
\hline Star Name & Frequency of occurrence of events (MHz) \\
\hline GJ 273 & 1066.652920 \\
\hline Teegarden's Star & $1066.652421,1066.678759,1333.316069$ \\
\hline Wolf 1061 & $1124.996688,1333.342057,1066.652726,1066.678878,1333.315905$ \\
\hline GJ 3323 & $1066.652808,1066.678803,1124.996748,1333.316017$ \\
\hline TRAPPIST-1 & $1375.003282,1066.678759,1066.652853,1333.316062$ \\
\hline $\mathrm{K} 2-3$ & $1124.996591,1333.342109,1066.678938,1066.652652,1333.315816$ \\
\hline $\mathrm{K} 2-72$ & $1066.652302,1066.679243,1333.315376,1333.342317$ \\
\hline Kepler-1649 & $1066.678610,1333.341870,1333.316270,1066.653442$ \\
\hline Kepler-560 & $1200.010400,1333.342005,1066.652808,1333.316002,1400.020804$ \\
\hline Kepler-186 & $1124.996584,1333.342161,1066.679027,1066.652548,1333.315697$ \\
\hline Kepler-438 & $1375.003260,1333.341938,1066.678707,1140.604001,1066.652913,1333.316144,1400.020640$ \\
\hline Kepler-296 & $1124.996450,1066.679258,1066.652294,1333.315361$ \\
\hline HD 180617 & $1124.996882,1333.341908,1066.678684,1333.316218,1066.652898$ \\
\hline GJ 96 & $1333.341774,1066.678498,1066.653151,1333.316442,1400.020275$ \\
\hline HD 69830 & $1124.996584,1333.342250,1066.679161,1066.652399,1333.315495$ \\
\hline BD-06 1339 & $1124.996793,1333.342005,1066.678818,1066.652793,1333.315987$ \\
\hline 16 Cyg B & $1066.652942,1066.678684,1333.316181,1400.020596$ \\
\hline HD 210277 & $1124.996584,1333.342206,1066.652466,1333.315570$ \\
\hline HD 19994 & $1333.342072,1066.678885,1066.652697,1333.315883$ \\
\hline HD 30562 & $1333.341781,1066.653062,1333.316330,1066.678483$ \\
\hline HD 82943 & $1124.996956,1333.341766,1066.678483,1333.316471$ \\
\hline HD 218566 & 1333.315503 \\
\hline kap CrB & $1066.678692,1124.996793,1333.316166$ \\
\hline HD 50554 & $1333.342176,1066.652525,1066.679042$ \\
\hline HD 22781 & $1375.003409,1124.996576,1333.342168,1200.010609,1066.679042,1066.652525,1333.315667,1400.021214$ \\
\hline HD 197037 & 1066.678699 \\
\hline HD 10697 & $1066.652257,1066.679280,1333.315317,1333.342340$ \\
\hline HD 111998 & $1124.996711,1066.652831,1066.678781,1400.020767,1333.316039$ \\
\hline $\mathrm{K} 2-18$ & $1066.678975,1066.652607,1333.315756$ \\
\hline Ross 128 & $1066.652950,1333.341908,1066.678669,1333.316196$ \\
\hline GJ 9066 & $1375.003505,1333.342295,1066.679221,1066.652332,1333.315406$ \\
\hline $\mathrm{K} 2-155$ & 1066.652764, 1066.678841, 1124.996614, 1333.315957, 1333.342034, 1374.991227, 1400.020871 \\
\hline Gliese 486 & $1333.341938,1066.678707,1200.010318,1066.652920,1333.316144$ \\
\hline
\end{tabular}

